\title{
Ocorrência de Fungos Micorrízicos Arbusculares em resíduo da mineração de bauxita revegetado com espécies arbóreas
}

\author{
Ana Lucy Caproni ${ }^{1,5}$, Avílio Antônio Franco², José Rodolfo Dantas de Oliveira Granha ${ }^{3}$ e Edson Luiz Souchie ${ }^{4}$
}

Recebido em 30/05/2005. Aceito em 10/08/2006

\begin{abstract}
RESUMO - (Ocorrência de Fungos Micorrízicos Arbusculares em resíduo da mineração de bauxita revegetado com espécies arbóreas). Avaliou-se a ocorrência de fungos micorrízicos arbusculares (FMAs) em tanques contendo resíduo da mineração de bauxita cultivados com espécies arbóreas inoculadas com Glomus clarum Nicol. \& Schenck e Gigaspora margarita Becker \& Hall na fase de viveiro. Acacia holosericea A. Cunn. ex G. Don juntamente com Sesbania virgata (Cav.) Pers. ou uma mistura de várias espécies foram transplantadas em tanques contendo resíduo de bauxita. Coletaram-se amostras de resíduos em agosto/1998 (estação seca) e abril/ 1999 (estação chuvosa), extraíram e identificaram-se os esporos de FMAs. Determinaram-se a densidade dos esporos de FMAs, a densidade relativa, a freqüência de ocorrência de cada espécie por período de amostragem e o índice de abundância e freqüência (IAF). A densidade dos esporos e a diversidade das espécies de FMAs foram baixas sob ambas as coberturas. Um maior número de esporos de Glomus clarum, e alto IAF, foram detectados no substrato cultivado com Acacia holosericea e Sesbania virgata na época seca. Isto também ocorreu em ambas as áreas na época chuvosa. Não foram encontrados esporos de Gigaspora margarita em ambas as áreas, nas duas épocas. Independentemente da inoculação, verificaram-se esporos de Archeospora leptoticha (Schenck \& Smith) Morton \& Redecker, Entrophospora colombiana Spain \& Schenck, Acaulospora mellea Spain \& Schenck e Glomus macrocarpum Tulasne \& Tulasne em abundância.
\end{abstract}

Palavras-chave: Amazônia, diversidade, mineração, revegetação, esporos

\begin{abstract}
Arbuscular mycorrhizal fungi occurrence in bauxite mining residue planted to tree species). The establishment of arbuscular mycorrhizal fungi (AMF) was evaluated in two areas of bauxite mining residue planted to tree species inoculated with Glomus clarum Nicol. \& Schenck and Gigaspora margarita Becker \& Hall in the nursery phase. Acacia holosericea A. Cunn. ex G. Don and Sesbania virgata (Cav.) Pers. and a mixture of several tree species were transplanted to deposits of containing bauxite mining residue. In August, 1998 (dry season) and April, 1999 (rainy season) residue samples were collected and AMF spores extracted and identified. AMF spore density, relative density, frequency of occurrence of each AMF species per sampling period and abundance and frequency index (AFI) were estimated. Spore density and AMF diversity were low in both areas. A greater number of Glomus clarum spores and high AFI were found in bauxite residue grown to A. holosericea and S. virgata in the dry season. This was also detected in both areas in the rainy season. Spores of Gigaspora margarita were not detected in both areas and sampling periods. Independent of AMF inoculation, a large number of spores of Archeospora leptoticha (Schenck \& Smith) Morton \& Redecker, Entrophospora colombiana Spain \& Schenck, Acaulospora mellea Spain \& Schenck and Glomus macrocarpum Tulasne \& Tulasne was detected.
\end{abstract}

Key words: Amazon, diversity, bauxite mining, revegetation, spores

\section{Introdução}

O entendimento da dinâmica das associações micorrízicas tem aumentado nos últimos anos (Hartnett \& Wilson 2002) bem como o reconhecimento da importante função desta simbiose no desenvolvimento das plantas nos ecossistemas terrestres. Porém, informações sobre o estabelecimento dos FMAs em tanques de resíduos da mineração de bauxita, onde há excesso de alumínio e falta de nutrientes para o desenvolvimento inicial das plantas, são escassas.

Segundo Pfleger et al. (1994) e Francis \& Read (1994) em ecossistemas pobres em nutrientes, a introdução de plantas inoculadas com fungos micorrízicos arbusculares (FMAs), podem garantir maior precocidade das plantas ao permitir que espécies micotróficas encontrem menores dificuldades na absorção de $\mathrm{P}$, água e outros nutrientes, permitindo

\footnotetext{
1 Fundação Universidade Federal de Rondônia, Campus Rolim de Moura, Av. Norte Sul 7300, 78987-000 Rolim de Moura, RO, Brasil

2 Embrapa Agrobiologia, C. Postal 74505, 23890-970 Seropédica, RJ, Brasil (avilio@cnpab.embrapa.br)

3 Universidade Fundação Oswaldo Aranha, Av. Paulo Erlei Alves Abrilantes 1325, Três Poços, 27251-970 Volta Redonda, RJ, Brasil (centifix@yahoo.com.br)

${ }^{4}$ Centro Federal de Educação Tecnológica, C. Postal 66, 75901-970 Rio Verde, GO, Brasil (esouchie@yahoo.com.br)

5 Autor para correspondência: analucycaproni@yahoo.com.br
} 
assim uma evolução mais rápida para os demais estádios sucessionais. Os mesmos autores salientam ainda que a presença dos microrganismos inoculados pode ser benéfica se não houver fungos nativos eficientes, ou então se o P disponível apresentar baixa concentração e as plantas usadas na revegetação responderem positivamente aos FMAs. A utilização de mudas que receberam inóculos de FMAs em viveiros é o principal método de introdução desses fungos em solos degradados ou em substratos destituídos de matéria orgânica (Caproni et al. 2003). Este método parece ser mais efetivo se as mudas receberem inóculos de FMAs adaptados às condições edafoclimáticas, por terem um efeito positivo na sobrevivência e crescimento das plantas nas áreas em recuperação (Pfleger et al. 1994) até que a comunidade nativa da sucessão natural passe a atuar.

O sucesso da recuperação de áreas degradadas depende também da seleção de espécies vegetais rústicas, tolerantes aos períodos secos e à baixa fertilidade do solo, e capazes de produzirem grande quantidade de matéria orgânica e sementes viáveis (Carneiro et al. 1999). Franco et al. (1992) utilizaram uma tecnologia baseada no plantio de espécies de leguminosas arbóreas associadas a rizóbios e fungos micorrízicos arbusculares como forma de superar as deficiências de $\mathrm{N}$ e $\mathrm{P}$ em áreas degradadas. Essa tecnologia foi utilizada com sucesso no Estado do Rio de Janeiro, e no Estado do Pará sobre o subsolo exposto em áreas de mineração de bauxita em Porto Trombetas. Na região de Porto Trombetas as espécies vegetais que mais se destacaram foram Acacia mangium Willd., A. holosericea A. Cunn. ex G. Don, $A$. angustissima (Mill.) Kuntze, Pseudosamanea guachapele (Kunth) Harms, Albizia saman (Jacq.) F. Muell. e Sclerolobium paniculatum Vogel (Franco et al. 1995). Com a introdução das leguminosas em áreas degradadas, esses pesquisadores observaram um aumento no número de esporos e na diversidade das espécies de FMAs. Entretanto, em processo de revegetação semelhante em Porto Trombetas, sobre resíduos da mineração de bauxita onde as condições estressantes são mais acentuadas e há excesso de umidade no período de estabelecimento das plantas, não se tem informações sobre as comunidades de FMAs estabelecidas.

A revegetação de tanques de resíduos da mineração de bauxita, utilizando mudas que receberam inóculos de FMAs é uma prática de grande importância, pois auxilia e acelera os processos da cobertura vegetal (Franco et al. 1995). Os FMAs podem auxiliar no estabelecimento das mudas contribuindo para a absorção de $\mathrm{P}$, outros nutrientes e água aumentando a resistência à doenças (Hartnett \& Wilson 2002).

O objetivo deste trabalho foi avaliar a população de FMAs em tanques de resíduo de bauxita, revegetados com plantas que receberam inóculos de Glomus clarum Nicol. \& Schenck e Gigaspora margarita Becker \& Hall, em duas épocas distintas.

\section{Material e métodos}

O distrito de Porto Trombetas $\left(56^{\circ} \mathrm{W} 1^{\circ} 40^{\prime} \mathrm{S}\right)$ no município de Oriximiná está localizado na região oeste do Estado do Pará, $100 \mathrm{~km}$ a oeste da confluência do Rio Trombetas com o Rio Amazonas. De acordo com a classificação de Köppen, o clima regional é do tipo AW e segundo Ferraz (1993) é bem definido com estação seca e chuvosa. A precipitação média mensal de junho a novembro/1998 foi de $71,5 \mathrm{~mm}$ e de dezembro/1998 a maio/1999 foi de $257,1 \mathrm{~mm}$.

O resíduo da lavagem da bauxita é uma lama, com até $40 \%$ de sólidos, que é eliminada durante o processo de beneficiamento. No reservatório, com uma área de 25 ha e 3 a $5 \mathrm{~m}$ de profundidade, o resíduo sofre um adensamento, inicialmente por sedimentação das partículas e posteriormente sob seu próprio peso, liberando água (Lapa 2000). A revegetação pode ser efetuada no material ainda não consolidado ou consolidado (Franco et al. 1995).

$\mathrm{Na}$ avaliação da ocorrência de FMAs, foram coletadas amostras em um tanque de resíduo, em uma área $\left(200 \mathrm{~m}^{2}\right)$, revegetada com Acacia holosericea A. Cunn. ex G. Don e Sesbania virgata (Cav.) Pers. e outra $\left(200 \mathrm{~m}^{2}\right)$ revegetada com um plantio misto de várias espécies vegetais (Acacia mangium Willd., Senna reticulata (Willd.) H.S. Irwin \& Barneby, Senna sp., Chamaecrista flexuosa (L.) Greene, Sesbania virgata, Leucaena leucocephala (Lam.) de Wit, Cecropia sp., Acacia holosericea, Hydrochorea corymbosa (Rich.) Barneby \& J.W. Grimes, Dalbergia spruceana (Benth.) Benth., Acosmium nitens (Vogel) Yakovlev, Stryphnodendron guianense (Aubl.) Benth., Peltogyne venosa (Vahl) Benth., Sclerolobium paniculatum Vogel, Parkia pendula (Will.) Benth. ex Walp., Parkia oppositifolia Spruce ex Benth., Enterolobium schomburgkii (Benth.) Benth. e Ormosia excelsa Benth.). Ambos foram revegetados sem a reposição da camada superficial orgânica e estavam com um ano de idade. Para o plantio misto, as mudas foram produzidas com substrato de matéria orgânica proveniente da floresta 
primária e receberam inóculos de Glomus clarum Nicol. \& Schenck e Gigaspora margarita Becker \& Hall. Esse substrato também continha as espécies: Acaulospora foveata Trappe \& Janos, A. laevis Gerdemann \& Trappe, A. mellea Spain \& Schenck, A. morrowiae Spain \& Schenck, A. rehmi Sieverding \& Toro, A. scrobiculata Trappe, A. spinosa Walker \& Trappe, A. tuberculata Janos \& Trappe, Acaulospora sp. 1, Acaulospora sp. 2, Acaulospora sp. 3, Archeospora leptoticha (Schenck \& Smith) Morton \& Redecker, Entrophospora colombiana Spain \& Schenk, E. contingua Spain \& Schenck, E. kentinensis $\mathrm{Wu} \& \mathrm{Liu}$, Entrophospora sp. 1, Entrophospora sp. 2, Entrophospora sp. 3, Entrophospora sp. 4, Gigaspora gigantea (Nicol. \& Gerd.) Gerd. \& Tappe, G. margarita, G. rosea Nicol. \& Schenck, Gigaspora sp., Glomus caledonium (Nicol. \& Gerd.) Trappe \& Gerd., G. claroideum Schenck \& Smith, G. etunicatum Becker \& Gerdemann, G. fasciculatum (Thaxter) Gerd. \& Trappe emend. Walker \& Koske, G. formosanum Wu \& Chen, G. geosporum (Nicol. \& Gerd.) Walker, G. invermaium Hall, G. macrocarpum Tuslane \& Tuslane, G. magnicaule Hall, G. nanolumem Koske \& Gemma, G. reticulatum Bhattacharjee \& Mukerji, Glomus sp. 1, Glomus sp. 2, Glomus sp. 3, Glomus sp. 6, Glomus sp. 7 e Scutellospora heterogama (Nicol. \& Gerdemann) Walker \& Sanders. O plantio com $A$. holosericea e $S$. virgata foi realizado através de hidrossemeadura, com as sementes misturadas com inoculantes de rizóbio e de Glomus clarum e Gigaspora margarita.

Nas áreas em estudo foram coletadas, aleatoriamente, quatro amostras compostas constituídas de 10 subamostras, em agosto/1998 (período seco) e abril/1999 (início do período chuvoso). Em agosto/1998, no plantio constituído de $A$. holosericea e $S$. virgata foram coletadas duas amostras compostas devido à alta umidade do resíduo, impossibilitando o acesso em toda a área. As subamostras foram coletadas na profundidade de $0-20 \mathrm{~cm}$, na zona das raízes finas das plantas. As subamostras foram homogeneizadas para formar as amostras compostas, que foram secas à sombra e colocadas em sacolas plásticas e armazenadas a $10{ }^{\circ} \mathrm{C}$ até o seu processamento em laboratório.

A amostragem composta das amostras de resíduo tem se mostrado suficiente para a avaliação de ocorrência das espécies, e tem sido muito usada por pesquisadores em função do menor volume de trabalho em laboratório e por não inviabilizar as avaliações utilizando a análise estatística não paramétrica.
As amostras dos solos foram processadas no laboratório da Embrapa Agrobiologia em Seropédica, RJ, onde foi feita análise química, granulométrica, determinação da umidade (Tab. 1) e as avaliações dos FMAs.

De cada amostra retirou-se $100 \mathrm{~mL}$ de solo e fizeram-se as extrações dos esporos dos FMAs conforme Gerdemann \& Nicolson (1963), utilizando peneiras com malhas de 720 e $38 \mathrm{~mm}$, seguida por centrifugação em água e sacarose. Após a contagem, os esporos foram colocados em lâminas com álcool polivinil em lactoglicerol (PVLG) e quebrados, delicadamente sob a lamínula, para a exposição das paredes internas. Na mesma lâmina um segundo grupo de esporos foi montado com PVLG + reagente de Melzer (1:1), sob outra lamínula. Os resultados da reação de cor ao reagente de Melzer foram utilizados para caracterizar as paredes dos esporos, melhorando, em alguns casos, a visibilidade, especialmente daqueles esporos com paredes aderentes ou muitos finas. Os esporos foram então identificados e contados por espécie.

A identificação das espécies de FMAs das amostras coletadas em campo foi feita segundo Schenck \& Perez (1988) e descrição morfológica disponível na internet na página da International Culture Collection of Arbuscular Mycorrhizal Fungi (INVAM 2000). A interpretação das características taxonômicas foi feita mediante observações em microscópio óptico com iluminação de campo-claro e objetiva de imersão.

Estimou-se a densidade (D) através do número dos esporos de FMAs em $100 \mathrm{~mL}$ de solo e a densidade de cada espécie $\left(D_{i}\right)$, como sendo a relação entre o número de esporos de determinada espécie por $100 \mathrm{~mL}$ de solo. As diferenças nas densidades entre as épocas de amostragens e as áreas amostradas foram testadas usando o teste não paramétrico de Mann-Whitney (Noether 1983).

A utilização da prova não-paramétrica $U$ de Mann-Whitney trata-se de uma das mais poderosas provas não-paramétricas, e constitui uma alternativa extremamente útil da prova paramétrica $t$ (Siegel 1975).

Estimou-se a densidade relativa de cada espécie de FMA $\left(D_{\mathrm{i}}\right)$ em cada época de amostragem (seca e chuvosa) e para o período inteiro de amostragem (seco e chuvoso), como sendo o número de esporos de uma dada espécie em proporção ao número total de esporos de todas as espécies naquele período (Brower et al. 1990), como representada pela equação $\mathrm{DR}_{\mathrm{i}}=\mathrm{n}_{\mathrm{i}} / \sum \mathrm{n}$, onde $\mathrm{DRi}=$ densidade relativa da espécie $\mathrm{i} ; \mathrm{n}_{\mathrm{i}}=$ número total de esporos da espécie i, em $100 \mathrm{~mL}$ 
Tabela 1. Análise química, granulométrica e umidade das amostras de solo dos tanques de resíduo com plantios de mudas de Acacia holosericea A. Cunn. ex G. Don e Serbania virgata (Cav.) Pers. e plantio misto, com uma coleta em agosto/1998 (após um ano de plantio) e outra em abril/1999 (após um ano e sete meses de plantio), em Porto Trombetas, PA.

\begin{tabular}{|c|c|c|c|c|}
\hline \multirow[t]{2}{*}{ Variáveis } & \multicolumn{2}{|c|}{$\begin{array}{l}\text { Resíduo com plantio de } \\
\text { A. holosericea e } S \text {. virgata }\end{array}$} & \multicolumn{2}{|c|}{ Resíduo com plantio misto } \\
\hline & agosto/1998 & abril/1999 & agosto/1998 & abril/1999 \\
\hline $\mathrm{N}\left(\mathrm{g} \mathrm{dm}^{-3}\right)$ & 0,15 & 0 & 0,04 & 0,24 \\
\hline $\mathrm{CO}\left(\mathrm{g} \mathrm{dm}^{-3}\right)$ & 0,93 & 0 & 0,09 & 0,44 \\
\hline $\mathrm{pH}$ & 5,1 & 5,2 & 5,2 & 0 \\
\hline $\mathrm{Al}^{1++}(\mathrm{cmol} / 100 \mathrm{ml})$ & 0,2 & 0,1 & 0,0 & 0 \\
\hline $\mathrm{Ca}^{2+}\left(\mathrm{cmol}_{\mathrm{c}}^{\mathrm{c}} / 100 \mathrm{ml}\right)$ & 0 & 0 & 0 & 0 \\
\hline $\mathrm{Mg}^{2+}(\mathrm{cmol} / 100 \mathrm{ml})$ & 0 & 0 & 0 & 0 \\
\hline $\mathrm{P}\left(\mathrm{g} \mathrm{dm}^{-3}\right)$ & 3 & 1 & 2 & 0 \\
\hline $\mathrm{K}\left(\mathrm{g} \mathrm{dm}^{-3}\right)$ & 19 & 17 & 30 & 0 \\
\hline Areia total $\left(\mathrm{g} \mathrm{kg}^{-1}\right)$ & 130 & (1) & 220 & (1) \\
\hline Silte $\left(\mathrm{g} \mathrm{kg}^{-1}\right)$ & 210 & (1) & 170 & (1) \\
\hline Argila total $\left(\mathrm{g} \mathrm{kg}^{-1}\right)$ & 660 & (1) & 610 & (1) \\
\hline $\begin{array}{l}\text { Umidade }\left(\mathrm{g} \mathrm{kg}^{-1}\right) \\
\text { (1) Análise não realizada }\end{array}$ & 293,4 & 314,3 & 200,5 & 202,7 \\
\hline
\end{tabular}

de solo; $\Sigma=$ soma do número dos esporos de todas as espécies em $100 \mathrm{~mL}$ de solo.

A freqüência de ocorrência de cada espécie $\left(\mathrm{F}_{\mathrm{i}}\right)$ foi calculada, em cada época de amostragem (seca ou chuvosa), pela determinação da porcentagem de amostras em que cada espécie foi isolada, em relação ao número total de amostras (Brower et al. 1990), representada pela equação $\mathrm{F}_{\mathrm{i}}=\mathrm{j}_{\mathrm{i}} / \mathrm{k}$. onde: $\mathrm{Fi}=$ freqüência de ocorrência da espécie $\mathrm{i} ; \mathrm{ji}=$ número de amostras nos quais a espécie i ocorreu; $\mathrm{k}=$ número total de amostras de solo.

$\mathrm{O}$ índice de abundância e freqüência (IAF) foi calculado para cada espécie pela soma da freqüência relativa $\left(\mathrm{FR}_{\mathrm{i}}\right)$ e densidade relativa $\left(\mathrm{DR}_{\mathrm{i}}\right)$ de seus esporos para o período inteiro de amostragem (período seco + chuvoso), multiplicado por 100 , podendo variar de 0 a 200. Este índice é similar ao valor de importância, que é um índice calculado para plantas superiores, a partir da soma da densidade relativa e freqüência relativa de cada espécie segundo Koske \& Gemma (1997). A freqüência relativa de cada espécie de FMA $\left(\mathrm{FR}_{\mathrm{i}}\right)$ foi estimada, para o período inteiro das amostragens (seco e chuvoso), como a proporção da soma das freqüências para todas as espécies (Brower et al. 1990), representada pela equação $\mathrm{FR}_{\mathrm{i}}=\mathrm{f}_{\mathrm{i}} / \sum \mathrm{f}$. Onde: $\mathrm{FR}_{\mathrm{i}}=$ freqüência de ocorrência relativa de uma dada espécie $i ; f_{i}=$ freqüência de ocorrência de uma dada espécie i; $\Sigma f=$ soma das freqüências de todas as espécies.

A estimativa do IAF tem a vantagem de usar mais de uma medida de influência, e tem a desvantagem de dar igual peso e valores completamente similares para cada combinação de dois valores relativos (Brower et al. 1990). As espécies foram ordenadas em quatro categorias, baseada em sua contribuição na comunidade total de esporos segundo Koske \& Gemma (1997): nenhuma importância: $\mathrm{IAF}=0$; pouca importância: $0<\mathrm{IAF} \leq 10$; moderada importância: 10 $<\mathrm{IAF} \leq 30$; grande importância: IAF $>30$.

\section{Resultados e discussão}

O resíduo de bauxita apresenta pouca disponibilidade de nutrientes, tais como $\mathrm{P}, \mathrm{K}$ e N , alto teor de argila em relação à areia total, alta umidade (de 20 a 30\%) e pH em torno de 5 (Tab. 1). O processo de revegetação nessas condições é facilitado com a inoculação das mudas com microrganismos (Franco et al. 1995), os quais aliviarão os efeitos da deficiência de nitrogênio e fósforo (Milnes 1999). O pH, o Al e o $\mathrm{P}$ podem limitar a ocorrência de espécies de FMAs. Siqueira et al. (1989) observaram que esporos das espécies do gênero Acaulospora tenderam a ocorrer com maior freqüência em solos com $\mathrm{pH}$ menor que 6,5 enquanto que as de Gigaspora em menor freqüência e muitas das espécies de Glomus mostraram-se pouco tolerantes a acidez elevada e baixo teor de fósforo. A textura argilosa do solo pode, também, ser um fator limitante para o desenvolvimento de FMAs, pois existe uma tendência em haver menor concentração de esporos de FMAs em solos muito 
argilosos (Sierverding 1991). A disponibilidade de água tem um grande efeito na infecção das raízes pelos FMAs, sendo que as plantas e os fungos têm um nível ótimo de água no solo para obter o peso máximo da planta e o número abundante de esporos no solo. Quando a planta cresce em tamanho, mais carboidratos estão disponíveis para o crescimento e reprodução dos fungos, com um superumedecimento, o crescimento da planta cai e a reprodução dos fungos também (Nelsen 1987). Além disso, os FMAs são aeróbios e necessitam de suprimento de $\mathrm{O}_{2}$ para seu metabolismo (Paula \& Siqueira 1987).

Tanto a densidade de esporos quanto o número de espécies de FMAs não diferiram significativamente entre as épocas de coleta no plantio misto (Tab. 2). Os poucos esporos encontrados no resíduo deve ser conseqüência da multiplicação das espécies inoculadas e daqueles presentes no substrato, utilizado para a produção de mudas, proveniente da floresta primária. Já, na área plantada com Acacia holosericea e Sesbania virgata houve maior densidade dos esporos na época seca (agosto/1998), o que está de acordo com os resultados encontrados em áreas florestais no período seco (Guadarrama \& Alvarez Sânchez 1999). Os autores relacionaram esses fatos com a fenologia das plantas, observando que o número de esporos aumenta quando as raízes diminuem sua taxa de crescimento (estação seca) e que o micélio se desenvolve intra e extra radicularmente durante a estação chuvosa, sugerindo que a umidade favorece a germinação dos esporos, resultando em alta colonização e baixa produção de esporos.

Em ambas as áreas de resíduo o número de espécies de FMAs nativos foi muito baixo em relação ao encontrado na floresta primária (Caproni et al. 2003), sendo encontradas 26 espécies na época seca e 17 na úmida. No tanque de resíduos de bauxita foram identificadas um total de oito espécies na comunidade de FMAs, sob plantio misto e plantio com A. holosericea e $S$. virgata (Tab. 3). Dentre essas, o gênero Acaulospora apresentou quatro espécies, seguido pelo gênero Glomus com duas espécies e Archeospora, Entrophospora e Scutellospora com uma espécie, cada gênero. O baixo número de espécies identificadas poderia estar relacionado às características do resíduo, principalmente com alto conteúdo de umidade e baixa porosidade. Pfleger et al. (1994) sugerem que as características edáficas, as espécies de plantas, a cobertura vegetal desde a época da revegetação e a disseminação dos propágulos interferem nas populações de FMAs. No entanto, ainda não ficou claro quais são os fatores que influenciam a esporulação, taxa de colonização e diversidade de FMAs em áreas degradadas e revegetadas.

Sob o plantio misto G. clarum apresentou maior número de esporos na época chuvosa e sob o plantio com A. holosericea e Sesbania virgata, esta espécie esporulou mais na época seca. No entanto, Gigaspora margarita, espécie inoculada, não foi detectada sob os plantios no tanque de resíduo (Tab. 3). Archeospora leptoticha esporulou somente no período seco (agosto/1998) no plantio com A. holoserecea e S. virgata. A. tuberculata e $S$. heterogama apresentaram maior esporulação na época chuvosa (abril/1999). O fato do aumento no número de esporos da maioria das espécies no segundo período de amostragem pode estar relacionado ao estabelecimento das mudas e expansão do sistema radicular, permitindo assim, maior formação das micorrizas. O efeito da umidade no desenvolvimento da micorriza, segundo Barea \& Jeffries (1995), é dependente dos isolados fúngicos envolvidos e das fases de desenvolvimento das plantas e também, em condições de campo, a umidade exerce diferentes efeitos sobre as espécies de FMAs (Klironomos et al. 1993).

Das espécies de FMAs identificadas no tanque de resíduo após um ano de plantio, seis ocorreram no período seco (agosto/1998) e seis no período chuvoso (abril/1999) (Tab. 4). Os esporos de Acaulospora tuberculata e Entrophospora colombiana foram

Tabela 2. Densidade de esporos e número médio de espécies de FMAs, em $100 \mathrm{~mL}$ de amostra de resíduo de bauxita sob plantio misto e plantio com Acacia holosericea A. Cunn. ex G. Don e Sesbania virgata (Cav.) Pers. em Porto Trombetas, PA.(1)

\begin{tabular}{lccccc}
\hline Áreas & \multicolumn{2}{c}{ Densidade de esporos } & & \multicolumn{2}{c}{ Número de espécies } \\
& agosto/1998 & abril/1999 & & agosto/1998 & abril/1999 \\
\hline Plantio misto & $154 \mathrm{a}$ & $290 \mathrm{a}$ & & $6 \mathrm{a}$ \\
Plantio com $A$. holosericea e Sesbania virgata & $1071 \mathrm{a}$ & $210 \mathrm{~b}$ & & $2 \mathrm{~b}$ & $5 \mathrm{a}$ \\
\hline
\end{tabular}

${ }^{(1)}$ Médias seguidas pela mesma letra, na linha entre épocas, não diferem entre si a 5\% de probabilidade pelo teste não paramétrico de MannWitney. 
Tabela 3. Densidade de esporos de FMAs por espécie, das amostras de solo, dos tanques de resíduo com plantios de mudas de Acacia holosericea A. Cunn. ex G. Don e Serbania virgata (Cav.) Pers. e plantio misto, com uma coleta em agosto/1998 (após um ano de plantio) e outra em abril/1999 (após um ano e sete meses de plantio), em Porto Trombetas, PA.

Espécies

$\frac{\frac{2 \text { Plantio misto }}{\text { agosto/1998 abril/1999 }}}{\%} \quad \frac{\frac{\text { A. holosericea e } S \text {. virgata }}{\text { agosto/1998 abril/1999 }}}{\%}$

Acaulospora mellea Spain \& Schenck

A. scrobiculata Trappe

A. tuberculata Janos \& Trappe

Archeospora leptoticha (Schenck \& Smith) Morton \& Redecker

Entrophospora colombiana Spain \& Schenck

Glomus clarum Nicol. \& Schenck

G. macrocarpum Tulasne \& Tulasne

Scutellospora heterogama (Nicol. \& Gerdemann) Walker \& Sanders

$\begin{array}{rr}34 & 43 \\ 20 & 113 \\ 31 & 0 \\ 0 & 8 \\ 3 & 0 \\ 34 & 121^{*} \\ 32 & 0 \\ 0 & 4\end{array}$

0

102

0

0

0

76

29

3

* Significativo a 5\% de probabilidade pelo teste não paramétrico de Mann-Whitney, entre as épocas. 0 - Não detectado.

encontrados no período seco, mas não no chuvoso. Ao contrário, os esporos de Archeospora leptoticha e Scutellospora heterogama ocorreram no período chuvoso e não no seco. A. mellea apresentou maior freqüência de ocorrência no período seco. A. scrobiculata apresentou maior esporulação e maior freqüência de ocorrência no período chuvoso. Glomus clarum apresentou densidades de esporulação em torno de $40 \%$ tanto no período seco quanto no chuvoso, porém com uma freqüência de ocorrência $5 \%$ maior no período chuvoso. É comum observar que a esporulação de algumas espécies de FMAs são sensíveis à situações de estresse hídrico. Segundo Sieverding (1991), as variações climáticas, caracterizadas pelo excesso de chuvas ou falta delas, ao longo do ano podem afetar a infectividade, o comprimento da raiz colonizada e o número de esporos.
Os FMAs, G. clarum e A. scrobiculata apresentaram alto valor de importância nos dois plantios (Fig. 1). G. macrocarpum apresentou elevado valor de importância em $A$. holosericea e $S$. virgata e $A$. mellea no plantio misto. Através deste índice sugere-se que G. macrocarpum, A. scrobiculata e G. clarum se destacam como espécies a serem consideradas em programas de revegetação, principalmente em tanques de resíduo de bauxita, restando observar sua eficiência simbiótica, pois estas espécies se adaptaram bem a estas condições ambientais adversas.

A interpretação da diversidade de FMAs através da contagem de esporos apresenta algumas dificuldades. A produção de esporos por uma determinada espécie pode variar com o estádio de desenvolvimento da planta hospedeira (Gemma et al.

Tabela 4. Densidade relativa $\left(\mathrm{DR}_{\mathrm{i}}\right)$ e freqüência de ocorrência $\left(\mathrm{F}_{\mathrm{i}}\right)$ das espécies de FMAs identificadas em amostra de resíduo de bauxita coletadas sob plantio misto de espécies arbóreas e plantio com Acacia holosericea A. Cunn. ex G. Don e Sesbania virgata (Cav.) Pers., com um ano de idade, em Porto Trombetas, PA.

\begin{tabular}{|c|c|c|c|c|}
\hline \multirow[t]{3}{*}{ Espécies } & \multicolumn{2}{|c|}{$\mathrm{DR}_{\mathrm{i}}$} & \multicolumn{2}{|c|}{$\mathrm{F}_{\mathrm{i}}$} \\
\hline & agosto/1998 & abril/1999 & agosto/1998 & abril/1999 \\
\hline & \multicolumn{2}{|c|}{$\%$} & \multicolumn{2}{|c|}{$\%$} \\
\hline Acaulospora mellea Spain \& Schenck & 22,28 & 15,03 & 50 & 25 \\
\hline A. scrobiculata Trappe & 2,87 & 43,14 & 33 & 38 \\
\hline A. tuberculata Janos \& Trappe & 4,46 & 0 & 17 & 0 \\
\hline Archeospora leptoticha (Schenck \& Smith) Morton \& Redecker & 0 & 1,60 & 0 & 13 \\
\hline Entrophospora colombiana Spain \& Schenck & 1,95 & 0 & 50 & 10 \\
\hline Glomus clarum Nicol. \& Schenck & 40,33 & 39,44 & 83 & 88 \\
\hline G. macrocarpum Tulasne \& Tulasne & 4,68 & 5,71 & 50 & 50 \\
\hline Scutellospora heterogama (Nicol. \& Gerdemann) Walker \& Sanders & 0 & 1,40 & 0 & 25 \\
\hline
\end{tabular}

0 - Não detectado. 


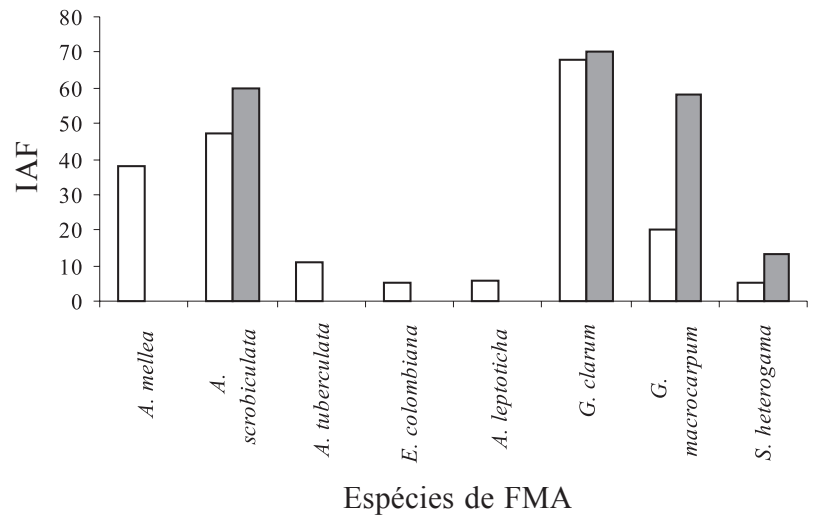

Figura 1. Índice de abundância e freqüência (IAF) das espécies de fungos micorrízicos arbusculares (FMAs) encontradas nos resíduos de bauxita e revegetados com plantio misto de espécies arbóreas e Acacia holosericea A. Cunn. ex G. Don e Sesbania virgata (Cav.) Pers. com um ano de idade, nas coletas de agosto/1998 e abril/1999, em Porto Trombetas, PA. ( $\square=$ Misto; - $=$. holosericea $+S$. virgata $)$.

1989; Smith \& Read 1997), requerendo várias amostragens durante a estação de crescimento (Stürmer \& Bellei 1994). Isto porque as mudanças sazonais e o estado fenológico da planta hospedeira influenciam a colonização micorrízica bem como a esporulação (Giovannetti 1985; Gould et al. 1996; Guadarrama \& Alvarez-Sânchez 1999). Entretanto, a área estudada apesar de apresentar duas estações definidas, uma chuvosa e outra seca, sem variações significativas de temperatura, durante os primeiros anos após o plantio, a superfície do rejeito permaneceu com alta umidade. Desta forma, os resultados de amostragens nos dois períodos podem refletir a comunidade de FMAs que esporulam nas várias fases do processo sucessional. Os resultados mostraram que o fungo micorrízico arbuscular G. clarum, quando inoculado, esporula no resíduo de bauxita sob plantio de espécies florestais tanto no período seco quanto no período chuvoso, enquanto que G. margarita não esporula nessas condições. As espécies, como A. leptoticha e E. colombiana, respondem à precipitação enquanto $A$. mellea e G. macrocarpum respondem à cobertura do solo na época do plantio. As espécies G. macrocarpum, A. scrobiculata e G. clarum devem ser consideradas em programas de revegetação, principalmente em tanques de resíduo de bauxita, por apresentarem maior esporulação nos períodos seco e chuvoso, dando uma indicação de boa adaptação à essas condições ambientais adversas.

\section{Agradecimentos}

À FAPERJ, ao CNPq e à Mineração Rio do Norte, pelo apoio financeiro; à Dra. Sandra B. Trufem, pela valiosa colaboração na taxonomia dos esporos de FMAs; ao Dr. José Carlos Polidoro, pela ajuda na análise estatística.

\section{Referências bibliográficas}

Barea, J.M. \& Jeffries, P. 1995. Arbuscular mycorrhizas in sustainable soil-plant systems. Pp. 521-559. In: A. Varma \& B. Hock (eds.). Mycorrhiza: structure, function, molecular biology and biotechnology. Berlim, Springer.

Brower, J.E.; Zar, J.H. \& Von Ende, C.N. 1990. Field and laboratory methods for general ecology. $3^{\text {rd }}$ ed. Dubuque, McGraw-Hill.

Caproni, A.L.; Franco, A.A.; Berbara, R.L.; Trufen, S.B.; Granha, J.R. \& Monteiro, A.B. 2003. Ocorrência de fungos micorrízicos arbusculares em áreas reflorestadas remanescentes da mineração de bauxita em Porto Trombetas, PA. Pesquisa Agropecuária Brasileira 38(12): 1409-1418.

Carneiro, M.A.C; Siqueira, J.O.; Curi, N. \& Moreira, F.M.S. 1999. Efeitos da inoculação de fungos micorrízicos arbusculares e da aplicação de fósforo no estabelecimento de forrageiras em solo degradado. Pesquisa Agropecuária Brasileira 34(9): 1669-1667.

Ferraz, J.B. 1993. Soil factors influencing the reflorestation on mining sties in Amazonia. Pp. 47-52. In: H. Lieth \& M. Lohman (eds.). Restoration of Tropical Forest Ecosystems. Dordrecht, Kluwer Academic Publishers.

Francis, R. \& Read, D.J. 1994. The contributions of mycorrhizal fungi to the determination of plant community structure. Plant and Soil 159: 11-25.

Franco, A.A.; Campello, E.F.C; Monteiro, E.M. \& Faria, S.M. 1992. Revegetação de solos degradados. Seropédica, EMBRAPA - Centro Nacional de Pesquisa Agropecuária Brasileira (Comunicado técnico, 9).

Franco, A.A.; Faria, S.M.; Dias, L.E.; Campello, E.F.C. \& Silva, E.M.R. 1995. Uso de leguminosas florestais noduladas e micorrizadas como agentes de recuperação e manutenção da vida do solo: um modelo tecnológico. Pp. 459-467. In: F.A. Esteves (ed.). Oecologia brasiliensis. Rio de Janeiro, UFRJ.

Gemma, J.N.; Koske, R.E. \& Carreiro, M. 1989. Seasonal dynamics of selected species of VA mycorrhizal fungi in a sand dune. Mycorrhizal Research 92: 317-321.

Gerdemann, J.W. \& Nicolson, T.H. 1963. Spores of mycorrhizal endogone species extracted from soil by wet-sieving and decanting. Transactions of the British Mycological Society 46: 235-244.

Giovannetti, M. 1985. Seasonal variations of vesiculararbuscular mycorrhizas and endogonaceous spores in a maritime sand dune. Transactions of the British Mycological Society 84: 679-684. 
Gould, A.B.; Hendrix, J.W. \& Ferriss, R.S. 1996. Relationship of mycorrhizal activity to time following reclamation of surface mine land in western Kentucky - 1: Propagule and spore population densities. Canadian Journal of Botany 74: 247-261.

Guadarrama, P. \& Álvarez-Sânchez, F.J. 1999. Abundance of arbuscular mycorrhizal fungi spores in different environments in a tropical rain forest, Veracruz, Mexico. Mycorrhiza 8: 267-270.

Hartnett, D.C. \& Wilson, G.W.T. 2002. The role of mycorrhizas in plant community structure and dynamics: lessons from grasslands. Plant and Soil 244: 319-331.

Klironomos, J.N.; Moutogolis, P.; Kendrick. B. \& Widden, P.A. 1993. Comparison of spatial heterogeneity of vesicular-arbuscular mycorrhizal fungi in two maple-forest soils. Canadian Journal of Botany 71: 1472-1480.

INVAM. 2000. International Culture Collection of Vesicular and Arbuscular Mycorrhizal Fungi. Species Description. Morgantown, West Virginia Agriculture and Forestry Experimental Station. Home page http:// invam.caf.wvu.edu (Acessado em: 07/2000).

Koske, R.E. \& Gemma, J.N. 1997. Mycorrhizae and sucession in plantings of beachgrass in sand dunes. American Journal of Botany 84: 118-130.
Lapa, R.P. 2000. A bauxita e o resíduo da bauxita. Pp: 25-36. In: R.L. Bozelli; F.A. Esteves \& F. Roland (eds.). Lago Batata: impacto e recuperação de um ecossistema amazônico. Rio de Janeiro, R.L. Bozelli.

Noether, G.E. 1983. Introdução à estatística: uma abordagem não paramétrica. $2^{\mathrm{a}}$ ed. Rio de Janeiro, Guanabara Dois.

Pfleger, F.L.; Stewart, E.L. \& Noyd, R.K. 1994. Role of VAM fungi in mine land revegetation. In: F.L. Pfleger \& R.G. Lingerman. Mycorrhizae and Plant Health. St. Paul, APS Press.

Schenck, N.C. \& Perez, Y. 1988. Manual for identification of vesicular-arbuscular mycorrhizal fungi. Gainesville, INVAM.

Siegel, S. 1975. Estatística não-paramétrica para as ciências do comportamento. São Paulo, Mc Graw Hill.

Sieverding, E. 1991. Vesicular-arbuscular mycorrhiza management in tropical agrosystems. Eschborn, GTZ.

Siqueira, J.O.; Colozzi Filho, A. \& Oliveira, E. 1989. Ocorrência de micorrizas vesicular-arbusculares em agro e ecossistemas do estado de Minas Gerais. Pesquisa Agropecuária Brasileira 24(12): 1499-1506.

Smith, S.E. \& Read, D.J. 1997. Mycorrhizal symbiosis. $2^{\text {nd }}$ ed., San Diego, Academic Press.

Stürmer, S.L. \& Bellei, M.M. 1994. Composition and seasonal variation on spore populations of arbuscular mycorrhizal fungi in dune soils on the island of Santa Catarina, Brazil. Canadian Journal of Botany 72: 359-363. 